\title{
Biorefinery: an Efficient Way to Sustainable Development of Chemical Industry - a Special Issue for International Conference on Biorefinery (ICB 07) and the 5th International Conference on Separation Science and Technology (ICSST2007)
}

\author{
Tianwei Tan $\cdot$ Jian-He Xu
}

Published online: 12 December 2009

(C) Humana Press 2009

Scientific and commercial interests in biorefinery become increasingly strong due to continual increase of petroleum price and environmental pollution problems. People have to face great challenges of global warming, reduction of $\mathrm{CO}_{2}$ emission, and energy security. Biorefinery, in which renewable raw materials are more and more widely used to produce biofuels such as ethanol and biodiesel, biobased chemicals including fine chemicals and bulk chemicals (e.g., 1,3-propanediol), and biodegradable materials such as polylactic acid and polyhydroxy acid, is becoming more important in chemical industry and energy production than ever before. The first international conference on biorefinery was therefore held in Beijing from October 20 to 23, 2007.

About 480 participants from 28 countries including USA, Canada, Japan, Germany, and Denmark took part in the successful conference. There were seven sessions, including bioenergy, biomaterials, biobased chemicals, feed stock, platform technology, and applications of biorefinery. Some new developments in biorefinery were presented in this conference. At same time, 5th International Conference on Separation Science and Technology, including 350 participants from 12 countries, was held in Beijing from October 14 to 16, 2007.

For efficient utilization of renewables in biorefinery, there are different methods: (1) thermal chemical method such as pyrolysis of biomass for fuel, (2) chemical method such as production of poly lactic acid, and (3) biocatalytic methods including enzymatic biocatalysis such as cellulase treatment of cellulosic biomass and whole-cell biocatalysis including fermentation. So biorefinery is a multidisciplinary technology, in which physical methods, chemical conversion, and biocatalysis as well as bioconversion will be integrated to obtain high-efficiency, low-cost, and low-energy consumption. Biocatalysis, a key

T. Tan $(\bowtie)$

Beijing Key Laboratory of Bioprocess, Biorefinery Research Center of Ministry of Education, Beijing University of Chemical Technology, Beijing 100029, People's Republic of China e-mail: twtan@mail.buct.edu.cn

J.-H. Xu

Sate Key Laboratory of Bioreactor Engineering, East China University of Science and Technology, Shanghai 200237, People's Republic of China 
technology in biorefinery, can be used not only for production of biofuel such as ethanol and biodiesel but also for synthesis of biodegradable plastics such as polyesters.

New, highly efficient and low-cost separation and purification technologies are also very important for bioprocess development. So some papers on separation and purification are also included in this special issue.

It is our great honor to publish a special issue for the biorefinery conference in this distinguished journal. After peer-review, about 50 papers were accepted for the special issue, with various topics including pretreatment of feed stock, modification of raw materials, thermal conversion of cellulose materials, screening of highly selective enzymes, biocatalytic production of bulk chemicals, separation of products, and new methods for purification.

On behalf of the organizing committee of Biorefinery Conference 2007, we would like to express our sincere thanks to Dr. Ashok Mulchandani and Springer staff for giving us this great opportunity and helps during the edition process of this special issue. We also like to thank the Ministry of Science and Technology of China, National Natural Science Foundation of China, Natural Science Foundation of Beijing, UOP (USA), BASF, Novozymes, and Leading Science Technology Development Co. Ltd. (China) for their financial supports. 